\title{
Method of Equilibrium Density Matrix. Energy of Interacting Valence Electrons in Metal
}

\author{
Boris V. Bondarev* \\ Moscow Aviation Institute, Volokolamskoe Shosse, 4, 125871, Moscow, Russia \\ *Corresponding author: bondarev.b@mail.ru
}

Received March 12, 2015; Revised March 16, 2015; Accepted March 18, 2015

\begin{abstract}
In this article we apply the method of density matrices for the description of the equilibrium system of interacting electrons. Variational principle of the density matrices is used in the framework of the mean field method for research of systems of valence electrons in metals. We obtained the model Hamiltonian describing the behavior of interacting electrons, which describes all the properties of superconductors. Note that was using the Coulomb potential that acts between two electrons in the coordinate space.
\end{abstract}

Keywords: electron distribution, interaction of electrons, electron energy, the model Hamiltonian

Cite This Article: Boris V. Bondarev, "Method of Equilibrium Density Matrix. Energy of Interacting Valence Electrons in Metal." International Journal of Physics, vol. 3, no. 3 (2015): 108-112. doi: 10.12691/ijp-33-3.

\section{Introduction}

If the system under consideration is in contact with a reservoir of heat and is characterized by temperature, then strictly speaking, its description by means of the density matrix is the only correct one. In quantum mechanics there are known pure and mixed states of the system. In this case the mixed state of the system is carried out by means of the density matrix $[1,2,3]$.

Complete statistical description of a system consisting of $N$ identical particles, fermions, in quantum mechanics is given by statistical operator $\hat{\rho}^{(N)}$ which satisfies the normalization condition

$$
\operatorname{Tr}_{12 \ldots N} \hat{\rho}^{(N)}=N \text { ! }
$$

Using this operator, we can build a hierarchical sequence of operators $\hat{\rho}^{(1)}, \hat{\rho}^{(2)}, \ldots$, defined by the relation

$$
\hat{\rho}^{(n)}=\frac{1}{(N-n) !} \operatorname{Tr}_{n+1 \ldots N} \hat{\rho}^{(N)}
$$

where $n=1,2, \ldots, \mathrm{N}-1$. Although statistical operators of lower order give not full but reduced description of manyparticle systems, they are irreplaceable due to their simplicity in cases when we need to obtain practically useful formulas and expressions. All physical quantities under consideration characterizing the state of a macroscopic system can be expressed accurately or approximately through the operators $\hat{\rho}^{(1)}$ and $\hat{\rho}^{(2)}$ or only through the one-particle operator $\hat{\rho}^{(1)}$. This enables to perform the reduced description.
For a system in the state of statistical equilibrium, $\mathrm{N}$ particle statistical operator has the form

$$
\hat{\rho}^{(N)}=\frac{1}{Z} \exp \left(-\beta \hat{H}^{(N)}\right),
$$

where $\hat{H}^{(N)}$ is the Hamiltonian of the system; $\beta$ is inverse temperature: $\beta=1 / k_{\mathrm{B}} \mathrm{T} ; \mathrm{Z}$ - statistic sum:

$$
Z=\frac{1}{N !} \operatorname{Tr}_{12 \ldots N} \exp \left(-\beta \hat{H}^{(N)}\right) .
$$

Formula (1.3) is the only case when the many-particle statistical operator can be known.

The thermodynamic functions of the state for equilibrium system can be defined by means of the statistical sum by the Gibbs method. However, the calculation of the statistical sum for a system of interacting particles is a very laborious task which can be solved exactly only in some rare cases. Even approximate calculation of statistical sum is very difficult.

One-particle statistical operator can be found by the formula

$$
\hat{\rho}^{(1)}=\frac{1}{(N-1) !} \operatorname{Tr}_{2 \ldots N} \hat{\rho}^{(N)}
$$

But an operation $\operatorname{Tr}_{2 \ldots \mathrm{N}}$ is even more complex than the calculation of the statistical sum.

The question arises, if it is possible to use other methods (maybe approximate) for finding the operators $\hat{\rho}^{(1)}$ and $\hat{\rho}^{(2)}$ directly without involving operators of higher order. In fact, such methods exist.

First, the one-particle statistical operator $\hat{\rho}^{(1)}$ can be found separately, this case is called the mean-field method. 
Or it can be found together with the operator for an $\hat{\rho}^{(2)}$ equilibrium system from the variational principle. This principle is based on the properties of some thermodynamic quantities (such as free energy) to take their extreme values when the many-particle system is in a state of statistical equilibrium.

Second, statistical operator $\hat{\rho}^{(1)}$ can be found as the result of the solutions of a kinetic equation governing the evolution of a nonequilibrium system. This equation was obtained in $[4,5,6]$ under the assumption that the evolution of a many-particle system occurs as a random Markov process. In [7] we proposed variation method for finding equilibrium density matrices of the first and the second orders. This method can be regarded as a generalization of the method Hartree- Fock - Slater to the case of arbitrary systems of fermions in contact with a reservoir of heat. In this paper mean field method is applied to describe the behavior of conduction electrons in metals.

\section{The Energy and Entropy of the System of Fermions}

Short description of a system consisting of $N$ identical particles fermions can be carried out using one- and twoparticle density matrices

$$
\rho_{11^{\prime}}=\rho\left(\alpha_{1}, \alpha_{1}^{\prime}\right), \rho_{12,1^{\prime}, 2^{\prime}}=\rho\left(\alpha_{1}, \alpha_{2} ; \alpha_{1}^{\prime}, \alpha_{2}^{\prime}\right),
$$

Where $\alpha$ is system of quantum numbers that determine the state of one particle.

One-particle density matrix $\rho_{\square}\left(\alpha_{1}, \alpha_{1}^{\prime}\right)$ satisfies the normalization condition

$$
\sum_{\alpha} \rho_{\square}(\alpha, \alpha)=N
$$

where $\rho(\alpha, \alpha)$ is the probability of filling the state $\alpha$.

The exact expression of the internal energy of a system of identical particles can be written by means of the density matrix (2.1) in the form

$$
E=\sum_{11^{\prime}} H_{11^{\prime} \square} \rho_{11}^{\prime}+\frac{1}{2} \sum_{12,1^{\prime} 2^{\prime}} H_{12,1^{\prime} 2^{\prime} \square} \rho_{1^{\prime} 2^{\prime}, 12} .
$$

Here $\mathrm{H}_{11}$ and $\mathrm{H}_{12,1^{\prime}}{ }^{\prime}$ are matrix elements of oneparticle Hamiltonian $\hat{H}^{(1)}$ and the Hamiltonian $\hat{H}^{(2)}$ of the interaction of two particles, respectively:

$$
H_{11^{\prime}}=H\left(\alpha_{1}, \alpha_{1}^{\prime}\right), H_{12,1^{\prime} 2^{\prime}}=H\left(\alpha_{1}, \alpha_{2} ; \alpha_{1}^{\prime}, \alpha_{2}^{\prime}\right) \text {. }
$$

Given that the two-particle density matrix, referring to the system of fermions must be antisymmetric, we will write for it the following approximate expression

$$
\rho_{12,1^{\prime}{ }^{\prime}}=\rho_{11^{\prime}} \rho_{22^{\prime}}-\rho_{12^{\prime}} \rho_{21^{\prime}} \cdot
$$

The density matrix $\mathrm{H}_{12,12}$ of the Hamiltonian of the interaction of two particles must also be antisymmetric. Substituting the expression (2.5) in the formula (2.3) leads to the formula

$$
E=\sum_{11^{\prime}} \mathrm{H}_{11^{\prime} \square} \rho_{11}^{\prime}+\sum_{12,1^{\prime}{ }^{\prime}} H_{12,1^{\prime} 2^{\prime} \square} \rho_{11^{\prime}} \rho_{2^{\prime} 2} .
$$

which corresponds to the mean-field approximation.

The transition from the coordinate representation, in which Hamiltonians are usually given, to some $\alpha$ representation is performed using orthonormal system of wave functions $\varphi_{\alpha}(q)$, where $q \equiv\{\mathbf{r}, \sigma\} ; \mathbf{r}$ is radiusvector of a particle; $\sigma$ is a spin variable. Knowing these functions, matrix elements of Hamiltonians (2.4) can be calculated by the well-known formulas

$$
\begin{gathered}
H\left(\alpha, \alpha^{\prime}\right)=\int \varphi_{\alpha}^{*} \hat{H}^{(1)} \varphi_{\alpha}^{\prime} d q, \\
H_{12,1^{\prime} 2^{\prime}}=\int \Phi_{12}^{*} \hat{H}^{(2)} \Phi_{1^{\prime} 2^{\prime}} d q_{1}, d q_{2},
\end{gathered}
$$

where the integral sign symbolizes the integration over the coordinates and the summation over the spin variable; $\Phi_{12}$ - Slater two-particle wave function:

$$
\Phi_{12}=\frac{1}{2}\left\{\varphi_{\alpha_{1}}\left(q_{1}\right) \varphi_{\alpha_{2}}\left(q_{2}\right)-\varphi_{\alpha_{1}}\left(q_{2}\right) \varphi_{\alpha_{2}}\left(q_{1}\right)\right\}
$$

Substituting this function into the formula (2.8), we obtain antisymmetric matrix

$$
\mathrm{H}_{12,1^{\prime} 2^{\prime}}=\frac{1}{4}\left(\mathrm{~V}_{12,1^{\prime} 2^{\prime}}-\mathrm{V}_{21,1^{\prime} 2^{\prime}}-\mathrm{V}_{12,21^{\prime}}+\mathrm{V}_{21,2^{\prime} 1^{\prime}}\right)
$$

where

$$
\begin{aligned}
& \mathrm{V}_{12,1_{2}^{\prime}}=\int \varphi_{\alpha_{1}}^{*}\left(\mathrm{q}_{1}\right) \varphi_{\alpha_{2}}^{*}\left(\mathrm{q}_{2}\right) \mathrm{U}\left(\mathrm{q}_{1}, \mathrm{q}_{2}\right) \\
& \cdot \varphi_{\alpha_{1}^{\prime}}\left(\mathrm{q}_{1}\right) \varphi_{\alpha_{2}^{\prime}}\left(\mathrm{q}_{2}\right) d \mathrm{q}_{1}, d \mathrm{q}_{2}
\end{aligned}
$$

$\mathrm{U}\left(\mathrm{q}_{1}, \mathrm{q}_{2}\right)$ is the potential energy of interaction between two fermions.

There is a representation in which the single-particle density matrix is diagonal, i.e. has the form

$$
\rho_{\mathrm{nn}}{ }^{\prime}=w_{\mathrm{n}} \delta_{\mathrm{nn}}{ }^{\prime}
$$

where $\mathrm{n}$ is the set of quantum numbers, which determines the state of one particle in a new representation; $w_{\mathrm{n}}-$ diagonal elements of the density matrix; $\delta_{n n}{ }^{\prime}-$ Kronecker characters. By definition, the value $w_{\mathrm{n}}$ is the probability that the state $\mathrm{n}$ is occupied by one of the particles. Thus, the function $w_{\mathrm{n}}$ describes the distribution of particles over states and satisfies the normalization condition

$$
\sum_{n} w_{n}=N
$$

The transition from n-representation to $\alpha$-representation that specifies the matrix elements (2.4) of Hamiltonians $\hat{H}^{(1)}$ and $\hat{H}^{(2)}$ is performed by a unitary transformation

$$
\rho\left(\alpha, \alpha^{\prime}\right)=\sum_{n} \Psi_{\alpha n}^{*} w_{n} \Psi_{\alpha^{\prime} n^{\prime}},
$$

where $\Psi_{a n}$ is a unitary matrix;

$$
\sum_{a} \Psi_{\alpha n}^{*} \Psi_{\alpha n^{\prime}}=\delta_{n n^{\prime}}
$$

Using the distribution function $w_{n}$, we can write the well-known expression for the entropy of a system of fermions: 


$$
S=-k_{B} \sum_{n}\left\{w_{n} \ln w_{n}+\left(1-w_{n}\right) \ln \left(1-w_{n}\right)\right\}
$$

This approximate expression is true only for the mean field method. The expression is accurate when electrons do not interact with each other.

\section{Variational Principle}

Referring to the formula (2.6), (2.14) and (2.16), we can say that free energy

$$
F=E-S T
$$

in the accepted approximation is a functional that depends on $w_{n}$ and $\Psi_{\alpha n}$. Since the equilibrium state of the system at fixed values of temperature and volume corresponds to the minimal free energy. Functions $w_{n}$ and $\Psi_{\alpha n}$ can be found by minimization of the free energy, taking into account conditions (2.13) and (2.15). Thus, we come to the problem of conditional extremum, to solve which by the Lagrange method we write a helper functional

$$
\Omega=E-S T-\mu \sum_{n} w_{n}-\sum_{n n^{\prime}}, \sum_{\alpha} \Psi_{\alpha n}^{*} \nu_{n n}{ }^{\prime} \Psi_{\alpha n^{\prime}},
$$

where $\mu$ and $v_{n n}$, are undefined multipliers.

Extremum conditions for the functional (3.2) lead to the equations for the distribution function $w_{n}$ and matrix $\Psi_{\alpha n}$ :

$$
\begin{gathered}
\ln \frac{1-w_{n}}{w_{n}}=\beta\left(\bar{\varepsilon}_{n}-\mu\right) \\
w_{n} \sum_{a^{\prime}} H_{\alpha \alpha^{\prime}}^{(e f f)} \Psi_{\alpha{ }^{\prime}}=\sum_{n^{\prime}}, v_{n n}{ }^{\prime} \Psi_{\alpha n^{\prime}},
\end{gathered}
$$

where $\bar{\varepsilon}_{n}$ is mean energy of one particle:

$$
\begin{gathered}
\bar{\varepsilon}_{n}=\varepsilon_{n}+\sum_{n^{\prime}} \varepsilon_{n n}{ }^{\prime} w_{n}{ }^{\prime} ; \\
\varepsilon_{n}=\sum_{\alpha \alpha^{\prime}}, \square \Psi_{\alpha n}^{*} H_{\alpha \alpha^{\prime}} \Psi_{\alpha{ }^{\prime} n}
\end{gathered}
$$

is the kinetic energy of a particle,

$$
\varepsilon_{n n^{\prime}}=2 \sum_{12,1^{\prime} 2^{\prime}} \sum_{\alpha} \Psi_{\alpha_{1}{ }^{*}}^{*} \Psi_{\alpha_{2} n^{\prime}} H_{12,12} H^{\prime} \cdot \Psi_{\alpha_{1}{ }^{n}} \Psi_{\alpha_{2}{ }^{\prime}{ }^{\prime}}
$$

Is the interaction energy of two particles in states with quantum numbers $\mathrm{n}$ and $\mathrm{n}$,

$$
\varepsilon_{n n^{\prime}}=\varepsilon_{n n}^{\prime}
$$

$H^{(e f f)}{ }^{\prime}{ }^{\prime}$
$\alpha \alpha$ mean-field approximation:

$$
H_{\alpha \alpha^{\prime}}^{(e f f)}=H_{\alpha \alpha^{\prime}}+2 \sum_{1,1^{\prime}} H_{\alpha \alpha_{1}, \alpha^{\prime} \alpha_{1}^{\prime}} \rho_{\alpha_{1}^{\prime} \alpha_{1}} .
$$

The solution is significantly easier in case when the properties of the system enable to predict in which the representation the density matrix must be diagonal. In this case, it only remains to solve equation (3.3). If it turns out that electrons do not interact with each other $\left(\varepsilon_{n n}{ }^{\prime}=0\right)$, then equation (3.3) leads to Fermi - Dirac function.
Solution of equation (3.3) have some interesting features, which are connected with its nonlinearity and the nature of the dependence of kernel $\varepsilon_{n n}$, on quantum numbers $\mathrm{n}$ and $n$. The aim of this work is to obtain expressions for the energy of interaction of two particles.

\section{The Energy of the Electrons in the Crystal Lattice}

The arrangement of the atoms in a crystal can be described by specifying the underlying lattice of Brave and specifying the location of the atoms in a separate unit cell. We will determine the position of one of atoms in the unit cell with the help of the vector $\mathbf{R}$, and the location of all other atoms in the cell relative to the first - using vectora. Let $s$ be a set of quantum numbers characterizing the wave function of one of the states of an electron localized in the neighborhood of the atom, the position of which is determined by the vector $\mathbf{R}+\mathbf{a}$. Using the introduced notations, we write the orthonormal system of wave functions describing localized electron states in the form

$$
\varphi_{\alpha}(q) \equiv \varphi(r R a, \sigma \mid a, s)
$$

where $\alpha=\{R, a, s\}$ isa set of quantum numbers that determine the state of the electron in the crystal lattice. As these functions the Vanier functions are convenient to use. Using these functions, we can calculate the matrix elements of the Hamiltonians (2.7) and (2.10).

After that, according to the method proposed in the previous section, you can find the density matrix of equilibrium state of the system of electrons in a crystal. In this paper we will consider only some of the simplest types of Hamiltonians which with some precision model the interaction and behavior of conduction electrons in real metals.

We consider the cases, when in the unit cell there is only one atom $(\mathbf{a}=0)$, and assume that the matrix (2.7) and (2.11) for the valence electrons have the form

$$
H_{\alpha \alpha^{\prime}}=\varepsilon_{R-R^{\prime}} \delta_{s S^{\prime}}
$$

here $\delta_{s s}$, is the Kronecker symbol,

$$
V_{12,1^{\prime}{ }^{\prime}}=V_{R_{1} R_{2}, R_{1}^{\prime} R_{2}^{\prime}} \delta_{s_{1} s_{1}^{\prime}} \delta_{s_{2} s_{2}^{\prime}},
$$

where the parameter $s$ takes a finite number $G$ of different values;

$$
\begin{aligned}
& V_{R_{1} R_{2}, R_{1}^{\prime} R_{2}^{\prime}}=\int \varphi\left(r_{1}\right)\left(r_{1}+R_{1}-R_{1}^{\prime}\right) \cdot U\left(r_{1}-r_{2}+R_{1}-R_{2}\right) \\
& \varphi\left(r_{2}\right) \varphi\left(r_{2} R_{2}-R_{2}^{\prime}\right) d r_{1} d r_{2}
\end{aligned}
$$

$\varphi(\mathrm{r}-\mathrm{R})$ is averaged wave function describing an electron localized in the vicinity of the site $\mathbf{R} ; \mathrm{U}\left(r_{1}-r_{2}\right)$ is the potential energy of Coulomb repulsion between electrons. In this case, the equilibrium density matrix describing the conduction electrons, would be

$$
\rho\left(\alpha, \alpha^{\prime}\right) \equiv \rho_{R R^{\prime}}^{s s^{\prime}}=\rho_{R R^{\prime}} \delta_{s s^{\prime}}
$$


Using the formulas (2.6), (2.10), (4.1) and (4.3), after simple transformations we come to the following expression for the energy of electrons:

$$
E=G\left\{\begin{array}{l}
\sum_{R R^{\prime}} \varepsilon_{R-R^{\prime}} \rho_{R^{\prime} R} \\
+\sum_{\{R\}} H_{R_{1} R_{2}, R_{1}^{\prime} R_{2}^{\prime}} \rho_{R_{1}^{\prime} R_{1}} \rho_{R_{2}^{\prime} R_{2}}
\end{array}\right\}
$$

Where $\{R\}=R_{1}, R_{2}, R_{1}^{\prime}, R_{2}^{\prime}$;

$$
H_{R_{1} R_{2}, R_{1}^{\prime} R_{2}^{\prime}}=\frac{1}{4}\left\{\begin{array}{l}
G\left(V_{R_{1} R_{2}, R_{1}^{\prime} R_{2}^{\prime}}+V_{R_{2} R_{1}, R_{2}^{\prime} R_{1}^{\prime}}\right) \\
\left.-V_{R_{2} R_{1}, R_{1}^{\prime} R_{2}^{\prime}}-V_{R_{1} R_{2}, R_{2}^{\prime} R_{1}^{\prime}}\right\}
\end{array}\right\} .
$$

If the electrons are distributed in lattice sites evenly, then the density matrix $\rho_{R R^{\prime}}$ can be represented in the form

$$
\rho_{R R^{\prime}}=\frac{1}{N_{L}} \sum_{k} w_{k} e^{i k\left(R-R^{\prime}\right)}
$$

where the summation is performed over the wave vectors $\mathbf{k}$, belonging to the first Brillouin zone; $N_{L}$ is the number of nodes in the grid; $w_{k}$ - electron distribution function on the wave vectors satisfying the normalization condition

$$
\frac{1}{N_{L}} \sum_{k} w_{k}=v
$$

$\mathrm{v}$ is the degree of zone occupation: $v=N / G N_{L}$.

Substitution of expression (4.7) in the formula (4.5) gives

$$
E=G\left\{\sum_{k} \varepsilon_{k} w_{k}+\frac{1}{2} \sum_{k k^{\prime}} \varepsilon_{k k^{\prime}} w_{k} w_{k}^{\prime}\right\},
$$

where $\varepsilon_{k}$ is the kinetic energy of an electron:

$$
\varepsilon_{k}=\sum_{R} \varepsilon_{R} e^{-i k R}
$$

$\varepsilon_{k k^{\prime}}$ - energy of the interacting of two electrons with wave vectors $\mathbf{k}$ and $k^{\prime}$;

$$
\varepsilon_{k k^{\prime}}=\frac{2}{N_{L}^{2}} \sum_{\{R\}} H_{R_{1} R_{2}, R_{1}^{\prime} R_{2}^{\prime}} \cdot e^{\left\{i k\left(R_{1}^{\prime}-R_{1}\right)+i k^{\prime}\left(R_{2}^{\prime}-R_{2}\right)\right\}}(4.10)
$$

To determine the structure of the kernel (4.10) in functional (4.9), refer to the formula (4.3). Taking into account that among matrix elements (4.3) the greatest are diagonal elements that correspond $R_{1}^{\prime}=R_{1}$ and $R_{2}^{\prime}=R_{2}$, we write the approximate formula

$$
\begin{aligned}
& V_{R_{1} R_{2}, R_{1}^{\prime} R_{2}^{\prime}}=U_{R_{1}-R_{2}} \delta_{R_{1} R_{1}^{\prime}} \delta_{R_{2} R_{2}^{\prime}} \\
& +U_{R_{1}-R_{2}}^{(0)} \delta\left(R_{1}-R_{1}^{\prime}-R_{2}+R_{2}^{\prime}\right)
\end{aligned}
$$

where $U_{R_{1}-R_{2}}$ is the average energy of Coulomb interaction of two electrons localized at the sites $R_{1}-R_{2}$, and the second term approximates the off-diagonal elements.
Strictly speaking, the function $U^{(0)}$ in the formula (4.11) should depend not only on $R_{1}-R_{2}$, but also on $R_{1}-R_{1}^{\prime}$. Using formula (4.6), (4.9) and (4.10) and (4.11), we obtain the following approximate expression for the interaction energy of electrons:

$$
E_{\text {int }}=\frac{1}{2} G\left\{\begin{array}{l}
v U_{0} N-\sum_{k k^{\prime}} J_{k-k^{\prime}} w_{k} w_{k}^{\prime} \\
+\sum_{k} I_{k} w_{k} w_{-k}
\end{array}\right\}
$$

where

$$
\begin{gathered}
J_{k-k^{\prime}}=\frac{1}{N_{L}} \sum_{R} U_{R} e^{i\left(k-k^{\prime}\right) R} ; \\
I_{k}=\sum_{R} U_{R}^{(0)}\left(G-e^{-2 i k R}\right)
\end{gathered}
$$

In (4.12), the first term is the energy of the direct Coulomb interaction of electrons, which does not depend on the distribution function $w_{k}$. The following component of the sum is the exchange energy of electrons. The kernel $J_{k-k^{\prime}}$ in this sum is a positive function that takes the largest value at $k^{\prime}=k$ and rapidly decreases as $\left|k^{\prime}-k\right|$ increases because the Coulomb interaction is long-range. Because the exchange energy is negative, the behavior of the function $J_{k-k^{\prime}}$ determines the effective attraction between electrons with close values of the wave vectors. While the positive terms in the formula (4.12) containing values $I_{k}$, determine effective repulsion between electrons with wave vectors $\mathbf{k}$ and $k^{\prime}=-k$.

Mean energy of an electron, corresponding to (4.12), would be

$$
\bar{\varepsilon}_{k}=\varepsilon_{k}-\sum_{k} J_{k-k^{\prime}} w_{k}^{\prime}+I_{k} w_{-k}
$$

In the model of free electrons moving in a field of positive charge ions evenly distributed in space, the energy of an electron is described by the formula [8]

$$
\bar{\varepsilon}_{k}=\frac{(h k)^{2}}{2 m}-\frac{e^{2}}{2 \pi^{2}} \int \frac{w_{k^{\prime}} d k^{\prime}}{\left|k^{\prime}-k\right|^{2}},
$$

where $m$ and $e$ are the mass and charge of an electron. Both in the formula (4.15) and in the formula (4.16) kernel $\bar{\varepsilon}_{k}$ has one common feature. It takes the smallest value $k^{\prime}=k$, and the largest, with $\left|k^{\prime}=-k\right| \rightarrow \infty$.

Unfortunately, using the formula (4.15), it is not only impossible to analytically solve equation (3.3), but even in any depth to research it. Therefore we approximate the function (4.13) by expression

$$
J_{k-k^{\prime}}=J \delta_{k k^{\prime}}
$$

where $J$ is appositive constant, $\delta_{k k^{\prime}}$ is Kronecker symbol; and the value of (4.14) we assume not depending on the wave vector

$$
I_{k}=I>0 .
$$


In this case the formula (4.15) has the form

$$
\bar{\varepsilon}_{k}=\varepsilon_{k}-J w_{k}+I w_{-k}
$$

and any interaction energy would be

$$
\varepsilon_{k k^{\prime}}=-J \delta_{k-k^{\prime}}+I \delta_{k+k^{\prime}}
$$

Since in such approximation, the property $\bar{\varepsilon}_{k}$ described above is preserved, we can assume that associated with this property features of the distribution function $w_{k}$ will not change significantly.

Function (4.20) is the model Hamiltonian of the interaction of electrons. Putting in equation (4.19) the wave vector $k^{\prime}$ equal in magnitude and opposite in direction to the vector $\mathbf{k}$, we see that the electron energy $I w_{-k}$ will decrease, when $w_{-k}$ decreases. This means that the electron in the state $\mathbf{k}$ displaces an electron from the state $k^{\prime}=-k$.

\section{Fermi - Dirac function and the distribution of electrons over the wave vectors}

Equality (4.7) essentially is a unitary transformation, diagonalizing the density matrix. The equation (2.16) takes the form

$$
S=-G k_{B} \sum_{k}\left\{w_{k} \ln w_{k} \mid\left(1-w_{k}\right) \ln \left(1-w_{k}\right)\right\}
$$

Minimalizing free energy taking into account the conditions of normalization (4.8), we come to the integral equation for finding the distribution function $w_{k}$ of conduction electrons over the wave vectors

$$
\ln \frac{1-w_{k}}{w_{k}}=\beta\left(\bar{\varepsilon}_{k}-\mu\right)
$$

where $\bar{\varepsilon}_{k}$ is mean energy of an electron with wave vector k:

$$
\bar{\varepsilon}_{k}=\varepsilon_{k}+\sum_{k^{\prime}} \varepsilon_{k k^{\prime}} w_{k^{\prime}}
$$

Substituting function (4.19)in equation (5.2), we obtain a nonlinear equation

$$
\ln \frac{1-w_{k}}{w_{k}}=\beta\left(\varepsilon_{k}-J w_{k}+I w_{-k}-\mu\right) .
$$

The solution of this equation is considered in articles $[3,9,10,11]$.

\section{Conclusions}

The modern microscopic theory of superconductivity was developed by Bardeen, Cooper and Schrieffer [8]. But this theory is not clear how superconductivity affects on the distribution of the electron on wave vector and how appears the attraction between electrons.

The obtained in this work the model Hamiltonian depends on the wave vectors $\mathrm{k}$, characterizes the interaction between electrons and contains two components. The first the term is always positive and describes the repulsion between electrons with wave vectors equal in absolute value and opposite in direction. This term at low temperature leads to the formation of anisotropy in some region of wave vectors. In this area, all the wave vector form pairs $\mathbf{k}$ and $-\mathbf{k}$, in one of which the state of the electron is almost full and the other is almost empty. This term leads to the explanation of the phenomenon of superconductivity. The second term is always negative and describes the attraction of two electrons with the wave vectors equal to each other. It explains the existence of pairs of electrons flying in one direction, and the energy gap in the dependence of the mean energy of the electron on its kinetic energy. We received the equation, from which we can find the distribution of electrons over the wave vectors.

The model of electrons in metals used in the present work can be the basis for an alternative theory of superconductivity. This model significantly differs from the models used in the modern theory of superconductivity. Here, the superconductivity is caused by the repulsion between electrons with the wave vectors $\mathbf{k}$ and $\mathbf{- k}$, and the energy gap in the spectrum is created due to the attraction between electrons with identical wave vectors $[9,10,11]$.

\section{References}

[1] J. von Neumann, Mathematical Foundations of Quantum Mechanics, Nauka, Moscow, 1964.

[2] K.Blum, Density Matrix Theory and Applications, Mir, Moscow, 1983.

[3] B.V. Bondarev, Density matrix method in quantum theory of cooperative process, Sputnik+, Moscow, 2013, p. 621.

[4] G.Lindblad, On the Generators of Quantum Dynamical Semigroups, Commun. Math. Phys. 1976, v. 48: 2, p. 119-130.

[5] B.V. Bondarev, Quantum markovian master equation for system of identical particles interacting with a heat reservoir, Physisa A, 1991 , v. 176 , p. 366-386.

[6] B.V. Bondarev, Conclusion quantum the kinetic equation from the Liouville-von Neumann equation, TMP, 1994, № 1, p. 33-43.

[7] B.V. Bondarev, Quantum lattice gas. Method of density matrix, Physisa A, 1992, v. 184, p. 205-230.

[8] N. Ashcroft, N. Mermin, Solid State Physics, Mir, Moscow, 1979.

[9] B.V. Bondarev, On some peculiarities of electrons distribution function over the Bloch states, Vestnik MAI, 1996, vol. 3, No. 2, p 56-65.

[10] B.V. Bondarev, New theory of superconductivity. Method of equilibrium density matrix. arXiv: 1412.600822 Sep 2013

[11] B.V. Bondarev, Density matrix method in quantum theory of superconductivity, Sputnik+, Moscow, 2014, p. 88. 